\title{
Research of Measure-Control Information Collection and Processing System Construction Based on IFix
}

\author{
Yong Ming \\ CHENGDU RADIO and TV UNIVERSITY, Sichuan, Chengdu610051 \\ 1234567@163.com
}

Keywords: Measure-Control Information; Field Bus; Spectrum; Terminal Service; Independent Computing Architecture.

\begin{abstract}
The paper about the research of measure-control information collection and processing system construction based on iFix starts from the monitoring and analysis of general industrial test information collection. And it puts forward that we adopt the industrial production field measurement and control module matching up the transmission of the field bus to make the data be collected, that is to say, make the data with different bus structures be collected through Unix Engineer Station. After the format conversion it transfers to the corresponding database server and the web server (the terminal publishing server) via the network to achieve the monitoring and analysis processing of test information collection. In this way it can provide a complete set of feasible and convenient system solutions for the users which transfer to the corresponding equipment with monitoring and analyzing information under the industrial production environment and put it into operation in practice. According to their respective rights, the users can browse or control through a proprietary network on their own workstations.
\end{abstract}

\section{Introduction}

Facing the needs of modern large-scale enterprises, seeking the industrial field dynamic data acquisition and management system based on web net browsing can make the technology and management personnel know the operation condition of the production site very conveniently and intuitively, and timely. It is not only convenient to make the manager put forward better decisions for enterprise macroscopic operation, but also make the engineering and technical personnel know the operation status of the production site and adjust the production parameters timely so as to provide a powerful guarantee for the safe and efficient production of the enterprise.

It can make many measure-control informations on the industrial field don't need to go to the site to be obtained and be obtained fully with multiple acquisitions in different places like the past. In this way, we can not only obtain the measure-control data with every operation front timely, but also we can get the complex analysis and judgment on the general work terminal or platform conveniently. So it not only can improve efficiency and save costs for the production control and management of the enterprise but also can establish high-technology image for the large-scale modernized enterprise effectively.

\section{iFIX Configuration Software}

After the investigations and researches in many ways we decide to select iFIX configuration software of Intellution company with the version 2.6. Compared with the previous version, the iFix version 2.6 increases the operational flexibility of software. Now the hardware key can support USB and parallel port configuration at the same time. The enhanced authorization is now available for the users of parallel terminal server. And updated encryption methods can only access the nodes defined. The iFix2.6 allows to track iHistorian state at the same time, and establish the expressions of the data from the iHistorian acquisition. New hardware encryption keys match with each set of iFix 2.6 software. We must use new hardware encryption keys to operate iFix. The new hardware encryption keys provide the following functions:

- Be compatible with USB or parallel port configuration. 
- Achieve the function of the corresponding option by internal control.

- Electronic upgrade. No need to change the hardware encryption keys.

- Provide parallel authorization to the terminal server.

The iFix version 2.6 supports parallel authorization to the users of the terminal server. This function removes the traditional special authorization. That is to say, it makes iFix permanently be assigned to access only a few client-side machines. What' $s$ more, it can use the authorization flexibly in the parallel environment so as to allow any client-side machine with configuration to access iFix. The parallel users over the number will be hung. When a user disconnects, it will allow the next one to $\log$ in. The iFix version 2.6 provides node encryption function. In the default state, the iFixSCADA server accepts write requests from any remote node. This function is very useful for us to prevent unknown or unauthorized node from modifying the SCADA servers. In addition, a new form, HTC(History Collection), has been added to the task control dialog box. HTC form can work with new iHistorian. The form displays the status of the iHistorian, and indicates any overloaded number to iHistorian. A new form has been added to the dialog box of expression editor. We use this form to choose the data source directly from the label of iHistorian collector. iHistorian can have category list by node, label, description or collector type.

\section{The Statistical Characteristics Analysis of Continuous Testing Information Variable Time-window}

In addition that we require to achieve the lists and trend charts to reflect the real-time data with all kinds of field measure-control, we also require to make real-time analysis and estimation of measure-control information with continuous records. It need to edit the relevant procedures and make them be embedded into the aforementioned composed system. The analysis and estimation arithmetic functions which are realized by major programming are auto-correlation function and spectral density function, correlation analysis method, visual-window processing, spectrum calculation and so on.

The Auto-correlation Function. For a signal $x(t)$, it always affects the value behind the time interval $\tau$ to a certain extent. That is to say, $x(t+\tau)$ relies on $x(t)$, and we call that $x(t+\tau)$ and $x(t)$ are related. The values of a signal itself in different time are related, and they have the relation of auto-correlation. In all the completed industrial measure-control information collection and analysis processing system, most of the continuous signals are auto-correlation. The relationship between the future value $x(t+\tau)$ and present value $x(t)$ of a signal can adopt the auto-correlation function to describe. So it is very important for the analysis of auto-correlation function. The auto-correlation function of a signal is the time average value of the product between $x(t)$ and $x(t+\tau)$ (seen in Figure 1).

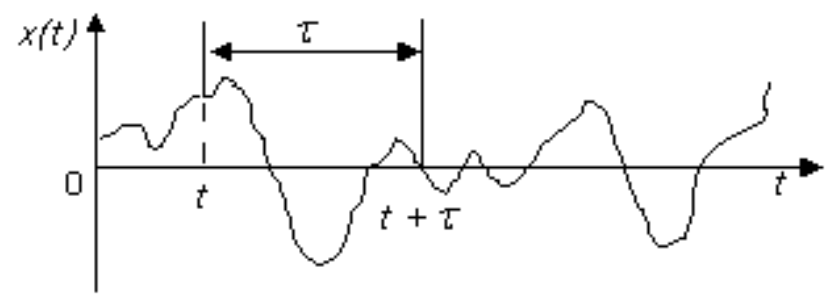

Figure 1 The measurement of auto-correlation

$$
\begin{aligned}
& R_{X X}(\tau)=\lim _{T \rightarrow \infty} \frac{1}{T} \int_{0}^{T} X(t)_{X}(t+\tau) d t \\
& =\lim _{T \rightarrow \infty} \frac{1}{T} \int_{0}^{T} X(t-\tau)_{X}(t) d t=E[X(t) \cdot X(t+\tau)]
\end{aligned}
$$




$$
\begin{aligned}
& =E\left[X(t-\tau)_{X}(t)\right]=E\left[X\left(t_{1}\right)_{X}\left(t_{2}\right)\right] \\
& =\int_{-\infty}^{+\infty} \int_{-\infty}^{+\infty} x_{1} \cdot x_{2} f\left(x_{1}, t_{1}, x_{2}, t_{2}\right) d x_{1} d x_{2}
\end{aligned}
$$

In the formula, $f\left(x_{1}, t_{1}, x_{2}, t_{2}\right)$ is joint probability density, $t_{2}-t_{1}=\tau$. The discrete calculation formula is

$$
X(k)=\frac{1}{N} \sum_{n=0}^{N-1} X(n) e^{-j(2 \pi / N) n k}
$$

Spectral Density Function. Fourier of auto-correlation function $R_{x x}(\tau)$ with signal $x(t)$ transforms to

$$
\begin{aligned}
& S_{X X}(j \omega)=\int_{-\infty}^{+\infty} R_{x X}(\tau) e^{-j \omega \tau} d \tau=\int_{-\infty}^{+\infty} R_{x x}(\tau)(\cos \omega \tau-j \sin \omega \tau) d \tau \\
& =\int_{-\infty}^{+\infty} R_{x x}(\tau) \cos \omega \tau d \tau=S_{x x}(\omega)
\end{aligned}
$$

Because $R_{x x}(\tau)$ is an even function, and the imaginary part in the formula equals to zero.Obviously the above integral is the real function of $\omega$. So we can use $S_{x x}(\omega)$ to represent. We call it as the power spectral density of the signal, spectral density, spectral density function and auto-power spectral density function. This is a mathematical definition.

Physical definition:The power in unit frequency range is power spectral density, namely

$$
G_{X X}(f)=\lim _{\Delta f \rightarrow 0} \frac{\psi_{x}^{2}(f, \Delta f)}{\Delta f}
$$

In the formula, $\psi_{x}^{2}$ is time average power in the random process,namely $\psi_{x}^{2}=E$. It spreads in the interval $(0, \mathrm{~T})$, and it also spreads in infrequency domain ( $0, \mathrm{f})$. It also uses accurate narrow band filter to filter sample function. The frequency of the filter is $\mathrm{f}$, and the bandwidth is $\Delta f$. And the signal power which goes through the filter is

$$
\psi_{x}^{2}(f, \Delta f)=\lim _{T \rightarrow \infty} \frac{1}{T} \int_{0}^{T} x^{2}(t, f, \Delta f) d t
$$

In the formula, $x(t, f, \Delta f)$ is the information in the frequency domain of $x(t)$ from $f$ to $f+\Delta f$. For the small $\Delta f$, power spectral density function can be defined as

$$
\psi_{x}^{2}(f, \Delta f) \approx G_{x x}(f) \Delta f
$$

More exactly, it is

$$
\begin{aligned}
& \quad G_{x x}(f)=\lim _{\Delta f \rightarrow 0} \frac{\psi_{x}^{2}(f, \Delta f)}{\Delta f}=\lim _{\Delta f \rightarrow 0} \frac{1}{\Delta f}\left[\lim _{T \rightarrow \infty} \frac{1}{T} \int_{0}^{T} x^{2}\left(t_{1} f_{1} \Delta f\right) d t\right] \\
& =\lim _{\substack{\Delta f \rightarrow 0 \\
T \rightarrow \infty}} \frac{1}{\Delta f \cdot T} \int_{0}^{T} x^{2}(t, f, \Delta f) d t
\end{aligned}
$$

$G_{x x}(f)$ is constant to be non-negative function with the real value.On the engineering, the applied estimation value is

$$
\hat{G}_{X X}(f)=\frac{1}{\Delta f \cdot T} \int_{0}^{T} x^{2}(t, f, \Delta f) d t
$$

In the formula, $f$ is center frequency. In this part, we need to use the discrete calculation formula to analyze it.

Correlation Analysis Method. The theoretical basis of correlation analysis method is Wiener-Hopf equation.Specifically we describe the equation below. 
We set the single input / single output system , $x(\mathrm{t})$ is the input variable of the system, and $y(\mathrm{t})$ is the actual measurement value of the output variable of the system.

$$
y(\mathrm{t})=Z(\mathrm{t})+C(\mathrm{t})
$$

Here $Z(\mathrm{t})$ is the real output of the system, and $C(\mathrm{t})$ is a random disturbance, which can be the measuring instrument error, and also can be the total error caused by the damage of linear assumption of the system and other various reasons.

According to the current theory of time-invariant systems, the output of the system can be written as the following form:

$$
Z(\mathrm{t})=\int_{0}^{\infty} g(\tau) x(t-\tau) d \tau
$$

In the formula, $g(\tau)$ is the impulse response function of the system. Obviously, if $g(\tau)$ is known, under the arbitrary input, the response of the system $\mathrm{Z}(\mathrm{t})$ can be immediately calculated by the formula above. Therefore, $g(\tau)$ describes the dynamic characteristics of the system completely. The system task of dynamic testing is to determine $g(\tau)$ a set of input - output record data(namely, the task of system identification). That is to say, we also use $\mathrm{x}(\mathrm{t})$ and $\mathrm{y}(\mathrm{t})$ from the set of data to establish and describe the system dynamic model $g_{m}(\tau)$. The mathematical model can be various, so $g_{m}(\tau)$ represents a model class. The actual impulse response of model and system can have some errors.

$$
y(\mathrm{t})=y_{\mathrm{m}}(\mathrm{t})+e(\mathrm{t})
$$

In the formula, $y_{\mathrm{m}}(\mathrm{t})$ is the output variable of the model, and $e(\mathrm{t})$ is the residual. In this kind of model $g_{m}(\tau)$, how can we find an optimal estimate $\hat{g}(\tau)$ of the system impulse response $g(\tau)$ ? We set the optimality criterion is that the result obtained can make the mean square value $J$ of the residual $e(\mathrm{t})$ be minimum.

$$
J=\lim _{T \rightarrow \infty} \frac{1}{T} \int_{0}^{T} e^{2}(t) d t
$$

That is to say, we use $\mathrm{J}$ as the minimum conditions to calculate $\hat{g}(\tau)$.

Assume that the input $\mathrm{x}(\mathrm{t})$ is ergodic stationary random process, and $C(\mathrm{t})$ is the random noise with $\mathrm{x}(\mathrm{t})$ statistical independence, the residual $e(\mathrm{t})$ can be written as

$$
e(\mathrm{t})=y(\mathrm{t})-y_{\mathrm{m}}(\mathrm{t})=y(\mathrm{t})-\int_{0}^{\infty} g_{m}(\tau) x(t-\tau) d \tau
$$

Then,

Therefore,

$$
\begin{aligned}
& e^{2}(t)=\left[y(t)-\int_{0}^{\infty} g_{m}(\tau) x(t-\tau) d \tau\right]^{2} \\
& =y^{2}(t)-2 y(\mathrm{t}) \int_{0}^{\infty} g_{m}(\tau) x(t-\tau) d \tau \\
& \quad+\int_{0}^{\infty} g_{m}(\tau) x(t-\tau) d \tau \int_{0}^{\infty} g_{m}(\lambda) x(t-\tau) d \tau
\end{aligned}
$$

$$
\begin{aligned}
J= & \lim _{T \rightarrow \infty} \frac{1}{T} \int_{0}^{T} y^{2}(t) d t-2 \int_{0}^{\infty} g_{m}(\tau) d \tau\left[\lim _{T \rightarrow \infty} \frac{1}{T} \int_{0}^{T} y(t) x(t-\tau) d t\right] \\
& +\int_{0}^{\infty} g_{m}(\tau) d \tau \int_{0}^{\infty} g_{m}(\lambda) d \lambda\left[\lim _{T \rightarrow \infty} \frac{1}{T} \int_{0}^{T} x(t-\tau) x(t-\lambda) d t\right]
\end{aligned}
$$

Seen from the definition about the correlation function of the ergodic process, the cross-correlation function of $x(\mathrm{t})$ and $y(\mathrm{t})$ is

$$
R_{x y}(\tau)=\lim _{T \rightarrow \infty} \frac{1}{T} \int_{0}^{T} y(t) x(t-\tau) d t
$$

The auto-correlation function of $\mathrm{x}(\mathrm{t})$ is 


$$
R_{x x}(\tau-\lambda)=\lim _{T \rightarrow \infty} \frac{1}{T} \int_{0}^{T} x(t-\lambda) x(t-\tau) d t
$$

Then

$$
J=R_{y y}(0)-2 \int_{0}^{\infty} g_{m}(\tau) R_{x y}(\tau) d \tau+\int_{0}^{\infty} g_{m}(\lambda) d \lambda \int_{0}^{\infty} g_{m}(\tau) R_{x x}(\tau-\lambda) d \tau
$$

\section{Conclusion}

The developed industrial measure-control information collection and analysis processing system has basically meet the requirements proposed at the beginning of design after the common acceptance of the user units and the related professors from University of Shanghai for Science and Technology. After the preoperation and operation the reliability, flexibility, easy operation and beautiful appearance and other aspects of the system have improved greatly than the previous similar system. And many aspects about the special functions have had large update and improvement. Up to now, the system has been running for nearly 12 months. And the system runs stably. Basically there is no any problem. And the system maintenance is also very convenient. The users have been able to get system maintenance independently. After the inquiries for many times we get the following feedback. The system runs stably, and the function gets the good use as the expect ion, and the whole system gets high praise from the users.

\section{Reference}

[1] A. N. Shilin,Yu. P. Bulatov,P. P. Bobkov,G. V. Lukin. Optical and electronic information and measuring system controlling the production of courses. Chemical and Petroleum Engineering,1993,2811:.

[2] M. E. Lomazov,L. B. Sul'povar,L. V. Besfamil'naya. Methodological problems of determining the economic effectiveness of standards for types and principal parameters of measuring instruments. Measurement Techniques,1973,158:.

[3] Samuel Rossel. Foveal fixation and tracking in the praying mantis. Journal of Comparative Physiology $\square$ A,1980,1394:. 